This document is the Accepted Manuscript version of a Published Work that appeared in final form in J. Chem. Phys. 125, 014308 (2006), copyright (C) American Institute of Physics after peer review and technical editing by the publisher. To access the final edited and published work see

$$
\text { jcp.aip.org }
$$




\title{
Excess Electron Localization Sites in Neutral Water Clusters
}

\author{
László Turi, a Ádám Madarász \\ Eötvös Loránd University, Department of Physical Chemistry, Budapest 112, P. O. Box \\ 32, H-1518, Hungary \\ Peter J. Rossky ${ }^{\mathrm{a}}$ \\ Department of Chemistry and Biochemistry, Institute for Theoretical Chemistry, \\ University of Texas at Austin, Austin, TX 78712-1167
}

We present approximate pseudopotential quantum mechanical calculations of the excess electron states of equilibrated neutral water clusters sampled by classical molecular dynamics simulations. The internal energy of the clusters are representative of those present at temperatures of $200 \mathrm{~K}$ and $300 \mathrm{~K}$. Correlated electronic structure calculations are used to validate the pseudopotential for this purpose. We find that the neutral clusters support localized, bound excess electron ground states in about $50 \%$ of the configurations for the smallest cluster size studied $(n=20)$, and in almost all configurations for larger clusters $(n>66)$. The state is always exterior to the molecular frame, forming typically a diffuse surface state. Both cluster size and temperature dependence of energetic and structural properties of the clusters and the electron

${ }^{a}$ E-mail: turi@chem.elte.hu, fax: (36)-1-372-2592. rossky@mail.utexas.edu, fax: (1)-512-471-3555. 
distribution are explored. We show that the stabilization of the electron is strongly correlated with the pre-existing instantaneous dipole moment of the neutral clusters, and its ground state energy is reflected in the electronic radius. The findings are consistent with electron attachment via an initial surface state. The hypothetical spectral dynamics following such attachment is also discussed. 


\section{Introduction}

Water cluster anions are among the most interesting charged species. ${ }^{1,2}$ The water cluster anions might also be considered as simpler models for the fully hydrated electron, ${ }^{3}$ bridging between the gaseous and the liquid phase species. Both the finite size cluster anions and the hydrated electron play an important role in a variety of physical processes. A relevant example, their role in electron-initiated processes in aqueous systems, has been recently reviewed. ${ }^{4}$

The satisfactory characterization of the water cluster anions has proved to be a challenge. Since the earliest experimental ${ }^{1,2,5}$ and theoretical works, ${ }^{6,7}$ the focus has been on the electronic structure and the related physical properties of the clusters. Theory has predicted two distinct types of cluster isomers: ${ }^{6,7}$ surface state clusters, where the cluster configuration supports a localized excess electron ground state with electron distribution outside the molecular frame, and clusters with compact interior states in a solvent void, more similar to those characteristic of the hydrated electron. ${ }^{8,9}$ On the other hand, experiments observed smooth transitions of the properties (vertical detachment energy, optical absorption spectra) from finite size clusters to the bulk hydrated electron. ${ }^{10,11,12,13}$ To add to the complex picture, simple models based on continuum dielectric theory indicated that both types of electron localization modes can be consistent with the experimental observations. ${ }^{7,14}$

The latest experimental ${ }^{15,16,17,18,19,20}$ and theoretical works ${ }^{21,22,23,24,25,26}$ provided additional insight into the cluster anion problem. A recent simulation study illustrated that, from an energetic viewpoint, the localization of the excess electron in a surface state 
or in an interior state depends on both the cluster size and the internal energy of the cluster. ${ }^{26}$ Smaller clusters preferentially stabilize the electron in a surface state. Surface state clusters can also be formed in larger clusters with increasing internal energy. The study also showed that the set of surface states and the set of interior states exhibit unique signatures in the simulation data, with that for the surface state clusters corresponding to the experimentally observed energetic, spectral, and structural trends with cluster size. These trends strongly supported assignment of surface bound states to the water cluster anions so far observed experimentally.

Beyond conditions of internal energy and cluster size, the experimental conditions of the formation of cluster anions can influence the observed state and physical properties. Neumark and co-workers measured the photoelectron spectra of larger water cluster anions $(n \leq 150)$ and attributed the spectral signatures to interior states. ${ }^{19,20}$ Varying the cluster temperatures, by observing clusters generated with both low and high backing pressures, they found a new feature in the colder (higher pressure) conditions with significantly smaller VDE. They interpreted the corresponding clusters as surface states. Nevertheless, the effect may have a kinetic, rather than an energetic basis ${ }^{20,27}$ with the new spectral features associated with differing hydrogen-bonding patterns ${ }^{17,18}$ in these surface state clusters.

Clearly, the character of the water cluster anions depends on several factors which are difficult to manipulate experimentally. Trivially, the physical properties change as the cluster grows. The temperature or internal energy dependence of the phenomena has also become evident lately. ${ }^{19,26}$ The role of the attachment process of the excess electron to neutral water clusters in the molecular jet, has not yet been quantitatively considered. 
What is the structure of the neutral cluster before the attachment occurs? What changes are initiated in the cluster structure by the electron attachment process? Once the electron is attached to the clusters, how do the clusters relax? These aspects would be expected to have a profound significance on the observable cluster properties including accessible relaxation processes. ${ }^{19}$ Usually, the formation of negative water clusters takes place via the attachment of slow secondary electrons to neutral water clusters. The initially formed cluster anions can then be stabilized by collisions, internal energy redistribution, and monomer evaporation. The molecular structure of the solvent in the remaining, potentially non-equilibrium, cluster anion may still retain a resemblance to the parent neutral ensemble, particularly for the initially coldest clusters.

In the present paper, we consider the simplest physical scenario, and investigate the electron localization sites on equilibrated neutral water clusters. This is equivalent to studying the initial conditions of low energy electron attachment to pre-existing equilibrium water clusters. Although it is unknown whether the experimental cluster distribution is at equilibrium, an equilibrium assumption may be used as a well defined working hypothesis in the absence of any specific alternative basis. Independent of its precise correspondence to experimental conditions, the assumption provides a reasonable framework to elucidate the attachment process under alternative characteristic conditions of internal energy or temperature. This approach is analogous to the experiments of Knapp et al. who performed electron attachment to presumably colder water clusters under collision-free conditions, and detected long-lived, more weakly bound water cluster anions. ${ }^{28,29}$ The attachment process is studied here by statistically analyzing the localization sites on neutral water clusters taken from equilibrium molecular dynamics 
trajectories. In particular, we examine whether there exist stable interior vs. exterior, surface, initial localization sites, and evaluate their relative importance. We also characterize the localization sites, both energetically and geometrically, and develop correlations between the relevant physical quantities. The dynamics of the initially formed cluster anions will be investigated in later work.

The structure of the paper is as follows. First, we briefly review the simulation method, and the electron-water pseudopotential used here. In Section III, we present and analyze the simulation results. The discussion focuses on both the size and the temperature dependence of geometric, energetic and spectroscopic properties. The comparison of the simulation results to similar analysis in bulk water is also given. Section IV concludes the paper.

\section{Methods}

We performed equilibrium classical molecular dynamics simulations for various size-selected water clusters $(n=20,30,45,66,104,200,500)$ in the microcanonical ensemble at energies consistent with a distribution at nominal $200 \mathrm{~K}$ and $300 \mathrm{~K}$ temperatures. The interaction between the water molecules are described by a three-site SPC potential with internal flexibility. ${ }^{30}$ While the SPC model is unlikely to correctly describe the details of water cluster energies precisely, particularly for smaller clusters, we focus here on relatively large clusters at relatively high internal energies, where the structure is expected to be analogous to liquid water, where the current model is adequate. The integration of the equations of motion is performed using the Verlet 
algorithm with 1 fs time step. ${ }^{31}$ The analyzed equilibrium trajectories are each of $200 \mathrm{ps}$ length.

Next, we consider the interaction of an excess electron with the equilibrium neutral water clusters. We select 500 such clusters, 400 fs apart, from the equilibrium trajectories. The interaction between the electron and the (classical) cluster is evaluated within the pseudopotential approach using an electron-water molecule pseudopotential. $^{32,33}$ This pseudopotential satisfactorily predicts both the energetics and the spectroscopic properties of an equilibrium ground state hydrated electron. ${ }^{33}$ Most recently, the pseudopotential has been successfully applied to rationalize previous experimental observations on water cluster anions. ${ }^{26}$ Several other successful applications indicate the reliability of the potential. ${ }^{34,35,36}$ Since, the pseudopotential has been optimized to reproduce the Hartree-Fock electron density of an electron-single water molecule system in the static exchange limit augmented by an "a posteriori" polarization potential, ${ }^{32,33}$ the present pseudopotential mainly neglects electron-molecule dispersion interactions. ${ }^{37,38}$ Thus, one might expect that the regime involving small electron-cluster interaction energies is somewhat distorted by the present pseudopotential, being too weakly bound. We shall examine this possibility below by comparing the computed vertical detachment energies (VDE) of an excess electron using the pseudopotential and a correlated ab initio method. The comparison is performed for thirty configurations of a test molecular dynamics run for the $\left(\mathrm{H}_{2} \mathrm{O}\right)_{12}^{-}$water cluster anion.

In the pseudopotential approach, the excess electron is treated in a plane-wave basis, and represented on $64^{3}$ evenly distributed grid points. The grid points span a $l_{\text {box }}=72.68 \AA$ long cubic box. The Schrödinger equation of the excess electron in the field 
of the classical water molecules is solved using an iterative and block Lanczos procedure described in the literature. ${ }^{39}$ In the MD test run for the $\left(\mathrm{H}_{2} \mathrm{O}\right)_{12}^{-}$cluster, the water molecules evolve under the combined influence from the other classical molecules and the electron (via the Hellman-Feynman theorem). ${ }^{39}$

For comparison, we have also performed electron localization site analysis in bulk liquid water at $300 \mathrm{~K}$. Although localization site distributions in liquid water have been statistically analyzed previously ${ }^{40,41}$ using a different pseudopotential, ${ }^{42}$ for the sake of consistency, we carried out similar calculations with the pseudopotential used here for the clusters. To generate configurations for the calculations, we have performed classical molecular dynamics simulation of 1600 water molecules in a cubic simulation cell using periodic boundary conditions. ${ }^{31}$ The electron-bulk water interactions have been calculated for 500 selected configurations of a 200 ps long equilibrium trajectory.

\section{Results}

Before the evaluation of the molecular dynamics runs for the neutral clusters, we tested the applicability of the present pseudopotential by comparing the computed VDEs to correlated $a b$ initio calculations at the MP2 level of theory. We note, that it is well established that performing correlated $a b$ initio calculations beyond second order perturbation theory MP2 becomes extremely difficult even for very small size clusters (above $n \approx 6$ ). Herbert and Head-Gordon performed benchmark calculations of VDEs for various conformers of small water cluster anions ( $n \geq 6)$, and evaluated the accuracy of second-order many-body perturbation theory. ${ }^{24,25}$ They found that MP2 theory is 
sufficient to reproduce VDEs within $30-50 \mathrm{meV}$ of the results from both experiment and coupled-cluster theory, with slightly underestimating the binding of the excess electron. These observations motivate the comparison below.

We carried out equilibrium molecular dynamics runs for $\left(\mathrm{H}_{2} \mathrm{O}\right)_{12}^{-}$cluster anions to generate cluster anion configurations for the comparison. Thirty cluster configurations were selected to calculate the excess electron binding energy using both the pseudopotential method and MP2 calculations. For the ab initio calculations we employed the $6-31(1+3+) \mathrm{G}^{*}$ basis set proposed by Herbert and Head-Gordon for smaller water cluster anions. The ab initio calculations have been performed using the Gaussian program package. ${ }^{43}$ The results are shown in Fig. 1.

In general, we find that the pseudopotential systematically underestimates the MP2 interaction energies by about $0.2 \mathrm{eV}$. This limitation most likely originates from the neglect of dispersion in the pseudopotential. Nevertheless, Figure 1 shows that the correlation is quite reliable. We note that the selected water configurations bind the excess electron with interaction energies in the 30-400 $\mathrm{meV}$ range (using the pseudopotential). Significantly larger clusters (n 45-200) were examined previously with the same pseudopotential. The results showed similar underestimation of VDEs (relative to experiment) for surface state anions, but the qualitative trends for the computed VDE and optical absorption spectra were in reasonably good agreement, with the experimental observations. ${ }^{26}$ These findings suggest that the pseudopotential based method can be used with confidence in a wide range of stabilization energies for qualitative purposes. We can also conlude, that, although the pseudopotential does not produce quantitative agreement with the $a b$ initio methods in the weak interaction energy 
regime, the good correlation between the pseudopotential and the MP2 results justifies its use for our qualitative statistical analysis on larger clusters. Nevertheless, it is noteworthy that the electron binding energy derived from the pseudopotential is, in general, underestimated for surface-bound electrons.

Now turning to the pseudopotential calculations for the larger neutral clusters, the first observation is that nearly all configurations stabilize the electron relative to the vacuum level. In particular, we first inspected whether the stabilized electron is, in fact, localized in the field of the neutral molecules, or just artificially bound by the finite basis size. Since the radius of gyration $\left(r_{\mathrm{g}}\right)$ of a uniform sphere of radius $r_{\mathrm{s}}$ is $r_{g}=\sqrt{\frac{3}{5}} r_{s}$, we defined a cutoff distance $r_{c u t}=\sqrt{\frac{3}{5}} \frac{l_{b o x}}{2}$ in terms of the length of the cubic grid box, $l_{b o x}$. The electron distributions with radius of gyration $\left(r_{e}=\left\langle r^{2}\right\rangle^{1 / 2}\right)$ larger than the cutoff distance were considered delocalized and ignored in the statistics. The configurations with localized electron distribution make up $50 \%$ of the total sample for $n=20$. The ratio gradually increases with the cluster size; it is $80-90 \%$ at $n=66$, while from $n=104$ practically all configurations have a localized electron distribution. We analyze only the localized cases here. It should be emphasized that the discarded cases are also mostly (very weakly) stabilized, but the finite basis effect would likely unphysically distort their properties. We do not rule out the possibility that these cases are actually diffuse, but bound states.

Figure 2 collects the probability distribution of the ground state energy of the excess electron attached to various size water clusters at $T=200 \mathrm{~K}$ and $T=300 \mathrm{~K}$ nominal temperatures. The distributions show a typical stabilization in the $0-400 \mathrm{meV}$ range. As 
the cluster grows, the interaction becomes gradually stronger, indicated by the maxima shifting to more negative energies with increasing cluster size. The average ground state energy as a function of cluster size (Figure 3) illustrates this effect. It is also evident, that the higher temperature clusters present structures that stabilize the excess electron somewhat more strongly. The strongest localization sites appear on the $n=500$ clusters at $300 \mathrm{~K}$ with $0.23 \mathrm{eV}$ average stabilization. These energies are in remarkable accord with the continuum model of Antoniewicz et al. that calculates the adiabatic electron affinity of a water droplet. ${ }^{45}$ They estimate $0.1 \mathrm{eV}$ stabilization for a surface state excess electron to a neutral water droplet of $10 \AA$ radius ( $n \sim 100-200$ water molecules, see Figure 3$)$. The qualitative shape of the distribution functions is similar at both temperatures, except that the $300 \mathrm{~K}$ distributions are, as would be expected, somewhat wider. We also note, that we tested clusters at $T=100 \mathrm{~K}$ temperature, but found similar behavior as for the $T=200 \mathrm{~K}$ case.

To assess the location of the excess electron relative to the molecular frame of the cluster, we use the radius of the cluster $\left(r_{\mathrm{c}}\right)$, the electron radius (radius of gyration, $r_{e}=\left\langle r^{2}\right\rangle^{1 / 2}$ ), and the distance between the centers of the electron and water distributions $(R)$. As discussed for equilibrium hydrated electron clusters,${ }^{26}$ an interior state is localized within the cluster, if $R+r_{\mathrm{e}}<r_{\mathrm{c}}$. For surface states, $R \sim r_{c}$, and significant electronic amplitude appears outside the cluster. For all the clusters examined, we found that the excess electron localizes outside the cluster in a relatively diffuse surface state. Not surprisingly, without relaxation, no interior state electronic distributions were observed in the examined samples of clusters. Although in the strongest binding configurations $(n=500)$ the radius of gyration of the excess electron approaches $4 \AA$, the 
typical radius is 7-17 $\AA$ depending on the cluster size. The size of the excess electron attached to neutral clusters is significantly larger than the radius in the corresponding (relaxed) hydrated electron clusters (3-6 A). ${ }^{26}$ Nevertheless, the increasing strength of the electron-cluster interaction is still reflected in the decreasing electron size as the cluster grows (Figure 4).

A transparent representation of the strong connection between the radius of gyration and the ground state electronic energy is given in Figure 5, which demonstrates the correlation between these two quantities at three cluster sizes and two temperatures. Although it is difficult to transform the data sets to linear form individually, we attempted to find a linear correlation between the average quantities at both temperatures. It turns out that the average ground state energy scales linearly with the average inverse square of the electron radius (with the slight deviation of the $n=500$ points) (Figure 6). Since the size of the electron is expected to reflect the size of the attracting potential well around the cluster, the correlation is in nice qualitative agreement with the simple particle-in-aspherical-box model.

The potential stabilizing the excess electron is mainly of electrostatic origin. This fact becomes apparent in the strong correlation between the total instantaneous dipole moment of the cluster and the stabilization of the electron (Figure 7). The linear $E_{0} \sim\langle\mu \mid\rangle$ relation is in clear accord with basic electrostatics. The geometric arrangement of the clusters is also consistent with the electron-cluster dipole interaction model. The cosine distribution of the orientation of the cluster dipole vector relative to the vector joining the centers of mass of the electron and the cluster indicates the tendency for the electron to concentrate in the direction of the cluster dipole (Figure 8). 
We now turn to the electronic excited states. The statistical analysis of the excited state distributions is a straightforward task. As can be expected, the excited electronic states are mostly unbound. There are exceptions; a significant number of water configurations exist which weakly stabilize the first excited state of the excess electron, as well. For the $n=500, T=300 \mathrm{~K}$ case, for example, the first excited state eigenvalue can be as low as $-0.3 \mathrm{eV}$. While the unoccupied excited states have no role in the adiabatic localization of the excess ground state electron, their dynamics is embedded in the dynamics of the optical absorption spectra. Since, the spectrum is predominantly determined by the energy gap distributions, the ground state-excited state energy gap distributions provide interesting information on the potentially observable spectroscopy of the nascent water cluster anions. Such a $t=0$ gap distribution for the $E_{0}-E_{1}$ difference is shown for the neutral $n=104$ water cluster at $300 \mathrm{~K}$ (Figure 9). As the cluster relaxes, the initial spectrum evolves in time to the equilibrium optical absorption spectrum. For comparison, we show here an equilibrium water cluster anion energy gap distribution for the same $n=104$ cluster at the same temperature (Figure 9) in a surface electronic state. ${ }^{26}$ Since the energy gaps for the neutral clusters supporting bound, localized electrons, are predominantly at very small values $(20-70 \mathrm{meV}$ at $300 \mathrm{~K})$, subsequent relaxation of the cluster anions would be characterized with a large blue shift of the maximum of the absorption spectrum. The maximum for the initial distribution function is located at around $50 \mathrm{meV}$ for $n=104$, while that of the relaxed cluster anion is computed to be at $0.78 \mathrm{eV}$ resulting in a sizeable Stokes-shift of approximately $0.7 \mathrm{eV}$ for the surface state relaxation. The width of the distribution also significantly increases upon relaxation, from less than $0.1 \mathrm{eV}$ to more than $0.4 \mathrm{eV}$. The situation, however, is further complicated by 
the fact that the electron could finally localize either on the surface or in the interior of the cluster. The different electron localization modes exhibit unique spectral signatures as was pointed out in a previous simulation study. ${ }^{26}$ It was predicted that smaller clusters stabilize the electron in surface states, while the interior states may become stable in larger clusters. For the $n=20-104$ surface states at $300 \mathrm{~K}$, one can, thus, predict a $0.5-0.8$ $\mathrm{eV}$ blue-shift, ending in a surface state, while for $n=200$ ending in an interior state, the shift would be enormous, approximately $2.0 \mathrm{eV}$. Nevertheless, one might expect a time scale separation between initial surface localization and formation of an interior state. Preliminary simulation results suggest that surface states may also exist in larger clusters, and the electronic energies of these surface states may approach those of the interior states for the same cluster.

At the end, it is instructive to compare the cluster localization sites to those in pure liquid water. Assuming that the properties of the initial interior localization sites in liquid water are transferable to clusters, one can directly contrast the two types of nascent distributions. We found that the ground state electronic energy in the bulk is almost always positive, with $0.38 \mathrm{eV}$ average. This value is $0.6 \mathrm{eV}$ higher, than the average stabilization for the surface states in the $n=500$ cluster $(-0.23 \mathrm{eV})$, and further illustrates the preference for the electron to localize in a weakly bound surface state in the cluster dipole direction. The radius of gyration of the initial interior electron in bulk water ( 8.4 $\AA$ ) is comparable to the size of the surface states in the largest clusters $(8.2 \AA, n=500$, $T=300 \mathrm{~K}$ ), as illustrated in Figure 4 by the dashed line. This size indicates significant degree of electron delocalization in liquid water. It is notable, that the computed ground state electronic energy is about $0.4 \mathrm{eV}$ deeper, and the radius is $2-3 \AA$ larger than 
calculated with a different pseudopotential. ${ }^{40}$ These differences, although reflecting the variation in the applied pseudopotentials, are not consequential.

\section{Discussion and Conclusions}

We have performed classical molecular dynamics simulations and subsequent quantum-mechanical analysis of the electron localization sites existing in various finitesize neutral water clusters. Correlated $a b$ initio test calculations on systems of twelve water molecules and an excess electron indicate that our pseudopotential approach provides a reasonable framework for the purpose of this study. Nevertheless, we conclude that the pseudopotential VDEs are usually underestimated relative to MP2 results for surface-bound electrons.

Analysis of the electron localization sites has been carried out from energetic, structural and spectroscopic viewpoints. We find that the interaction of the neutral water clusters and slow electrons (the usual condition in cluster anion experiments) leads to the formation of electronic states which are located on the surface of the molecular cluster. Bound, localized states occur in about half of the configurations of the smallest cluster size considered here $(n=20)$ and in practically all configurations for larger cluster $(n>66)$. The surface electronic states are diffuse, and significant amplitude of the wavefunction is located outside the molecular frame. Although these clusters bind the electron weakly, it is reasonable to assume that these structures serve as starting points to the formation of the fully relaxed water cluster anions. 
Two characteristic pathways can be conceived for the relaxation of the initially formed anions, one leading to interior electronic states, and the other to persistent surface electronic states. For smaller clusters, it was observed at $T \geq 200 \mathrm{~K}$ in quantum MD simulations ${ }^{26}$ that preformed interior states always relax to the surface state indicating that excess electrons in smaller clusters persist in surface states. However, the observation of stable interior states for larger clusters ${ }^{26}$ does not a priori exclude the possibility that large cluster surface states are also at least metastable. In fact, our preliminary simulations show that even nanoscale clusters $(n=500)$ can stabilize the electron on the surface. This observation implies that there may exist at least two freeenergy minima on the path of the excess electron from exterior state to the cluster core. A quantum umbrella sampling simulation study ${ }^{46}$ supports the claim that both types of electronic states can be local free energy minima, and the rate of exchange between them is size and temperature dependent. Whether the attachment and localization dynamics follows a sequential path, with initial formation of a surface polaron, is the subject for additional studies.

\section{Acknowledgment}

L.T. gratefully acknowledges the tenure of the Bolyai Research Fellowship. The work was supported by a grant to L. T. from the National Research Fund of Hungary (OTKA) under Contract No. T049715. P. J. R. is grateful to the National Science Foundation (CHE-0134775) and the R. A. Welch Foundation (F-0019) for support of this work 


\section{Figure Captions}

Figure 1. Correlation between the VDE of $\left(\mathrm{H}_{2} \mathrm{O}\right)_{12}^{-}$anions computed with a pseudopotential and ab initio MP2/6-31(1+3+) $\mathrm{G}^{*}$ method.

Figure 2. Probability distributions of the ground state energy of the excess electron attached to $n=20$ (dash-dot), 45 (dot), 104 (dash) and 500 (solid) neutral water clusters at $T=200 \mathrm{~K}$ and $300 \mathrm{~K}$.

Figure 3. The average ground state energy of the excess electron at $T=200 \mathrm{~K}$ (triangle) and $300 \mathrm{~K}$ (square).

Figure 4. The average radius of gyration of the excess electron $\left(r_{e}=\left\langle r^{2}\right\rangle^{1 / 2}\right)$ at $T=200 \mathrm{~K}$ (triangle) and $300 \mathrm{~K}$ (square). The dashed line shows the size of the nascent interior states in bulk water at $300 \mathrm{~K}$.

Figure 5. Correlation between the ground state energy and the size of the excess electron at $T=200$ and $300 \mathrm{~K}$ for $n=45,104$ and 500 clusters.

Figure 6. Average ground state energy for different size clusters as the function of the inverse square of the size of the excess electron at $T=200 \mathrm{~K}$ (triangle) and $300 \mathrm{~K}$ (square). Figure 7. Average ground state energy of the excess electron for different size water clusters as the function of the average instantaneous dipole moment of the cluster at 300 $\mathrm{K}$.

Figure 8. Probability distribution of the orientation of the cluster dipole relative to the electron for $n=20$ (dash-dot) , 45 (dot), 104 (dash) and 500 (solid) neutral water clusters at $T=200 \mathrm{~K}$ and $300 \mathrm{~K}$. 
Figure 9. Probability distribution of the energy gap of the excess electron in the completely relaxed anion $^{26}$ (dashed) and in the initially formed anion (solid) for $n=104$ at $T=300 \mathrm{~K}$. 
Figure 1. Turi, Madarász, and Rossky

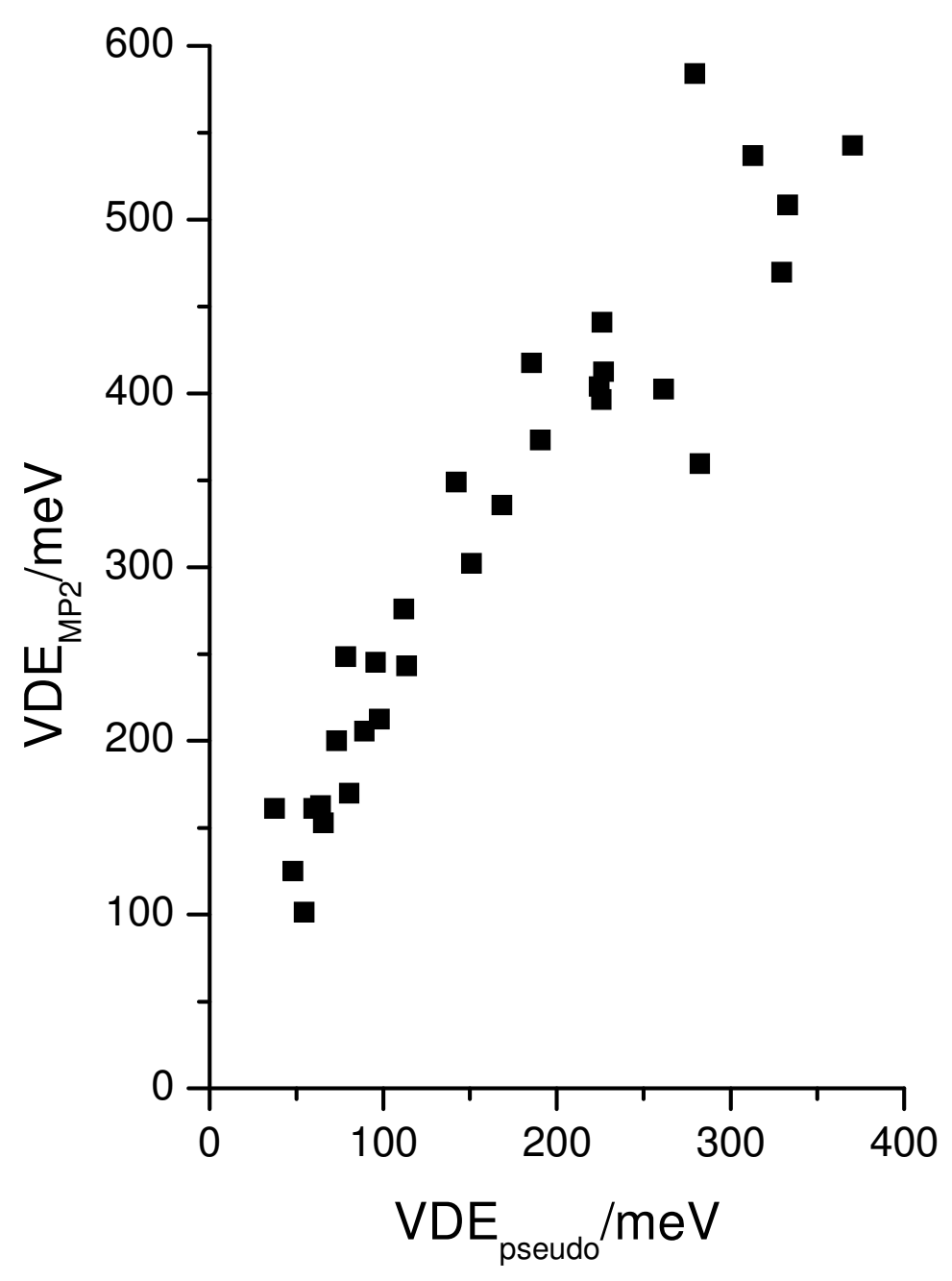


Figure 2. Turi, Madarász, and Rossky

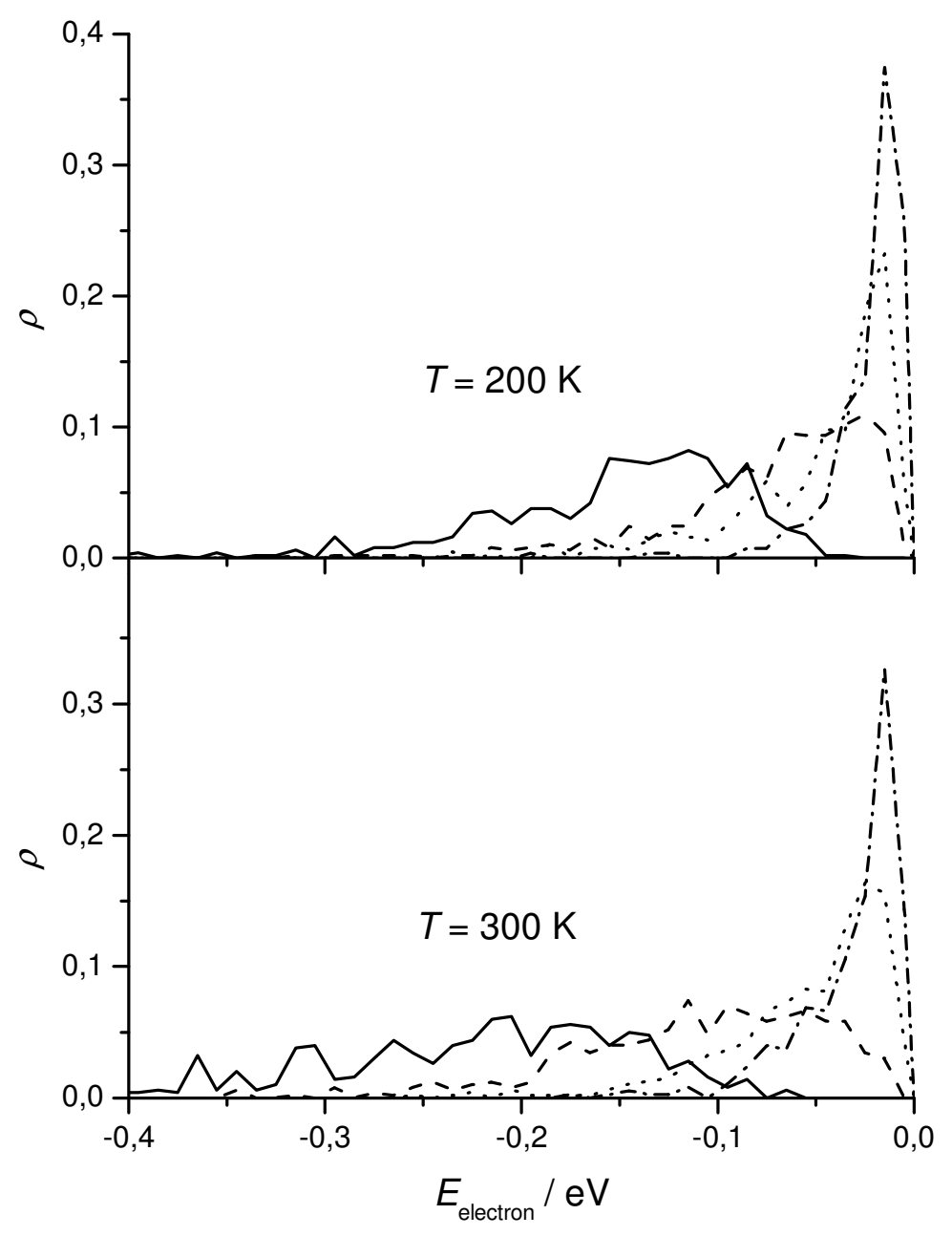


Figure 3. Turi, Madarász, and Rossky

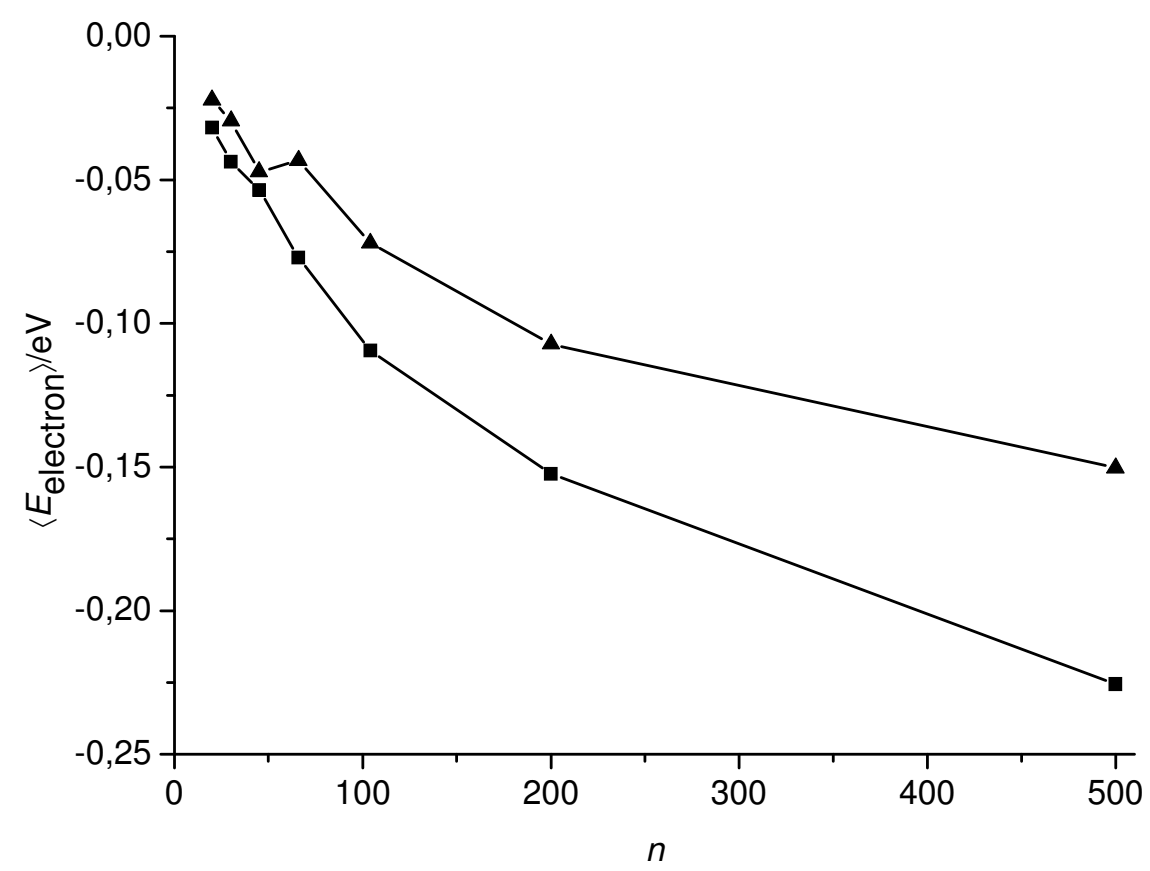


Figure 4. Turi, Madarász, and Rossky

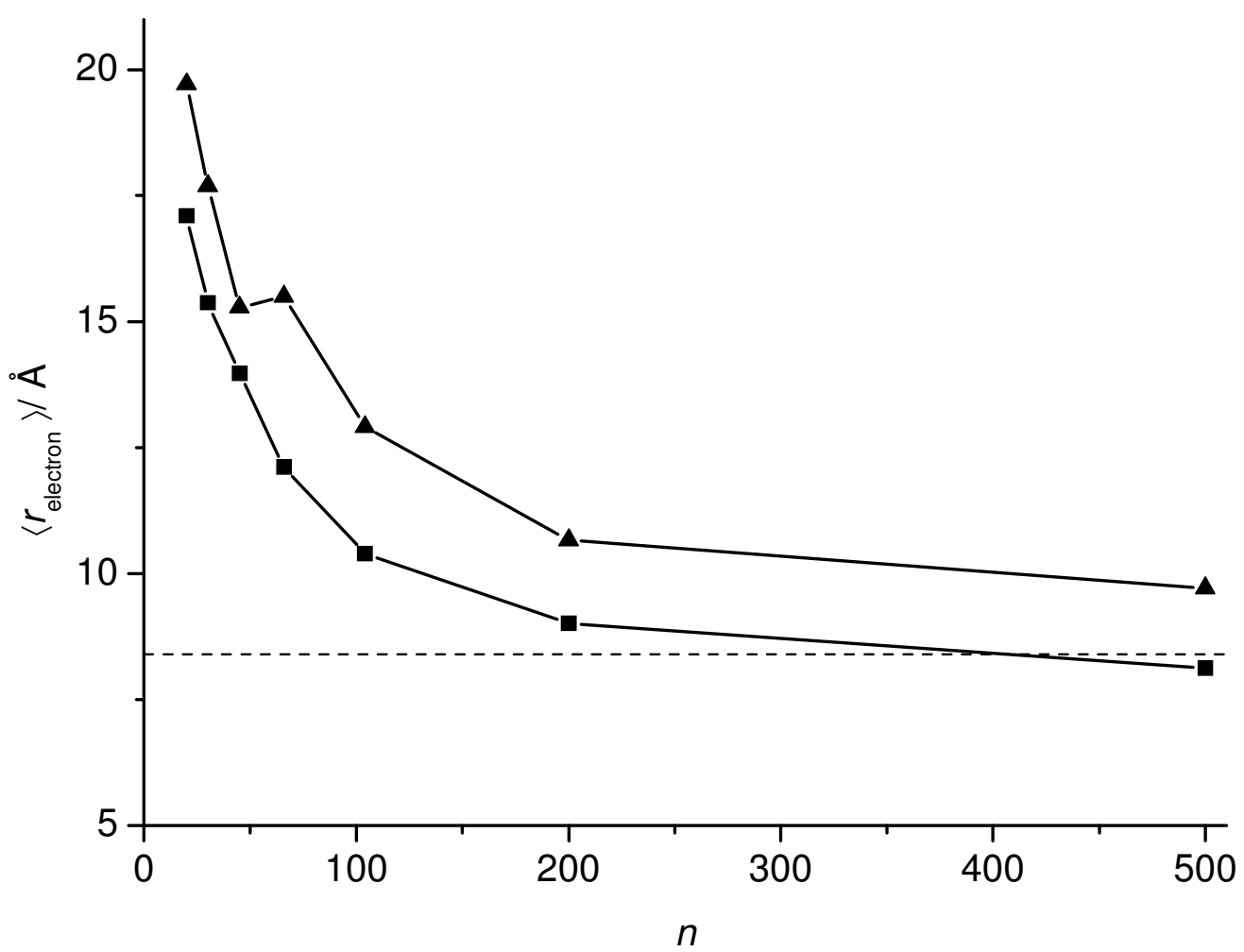


Figure 5. Turi, Madarász, and Rossky

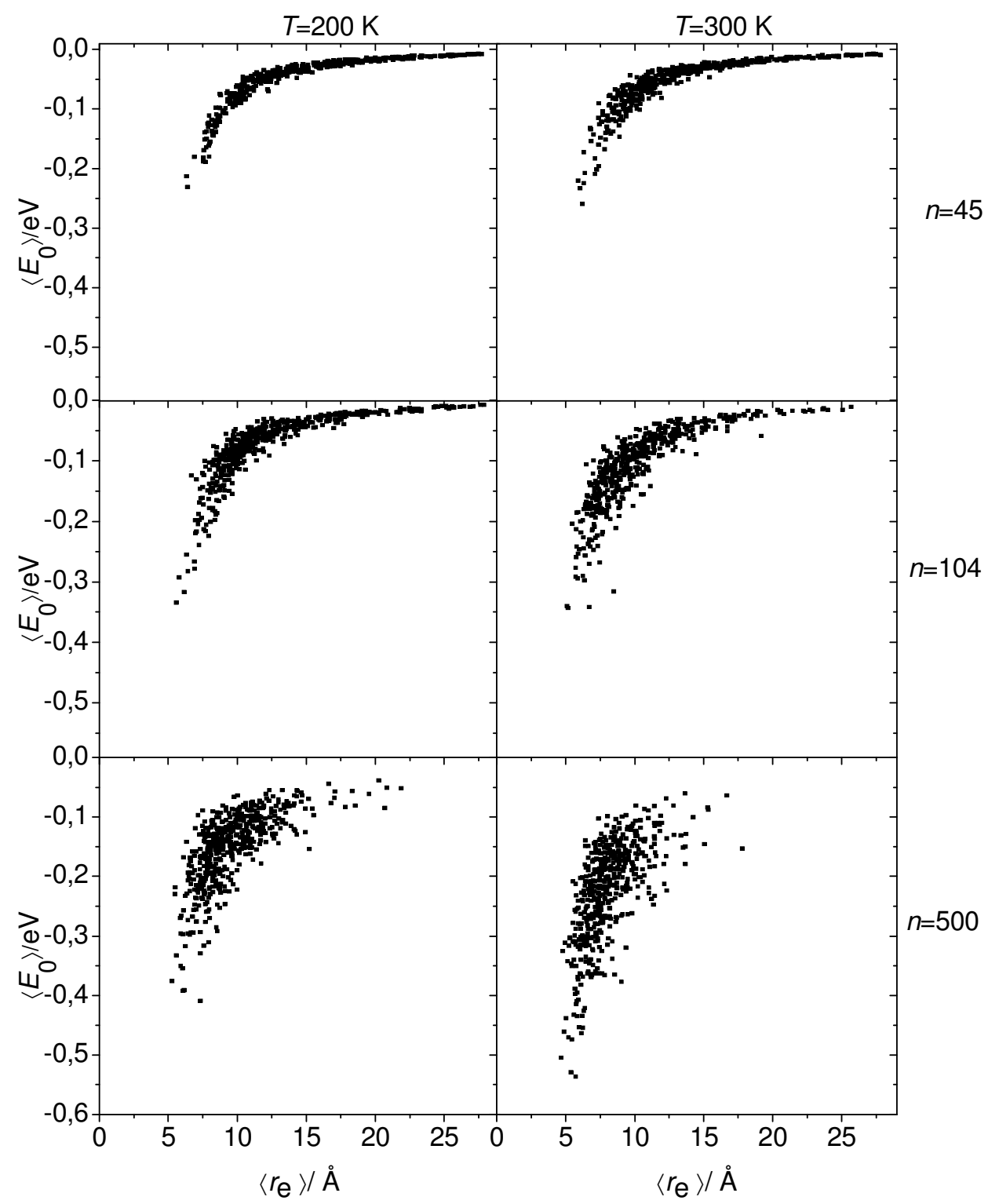


Figure 6. Turi, Madarász, and Rossky

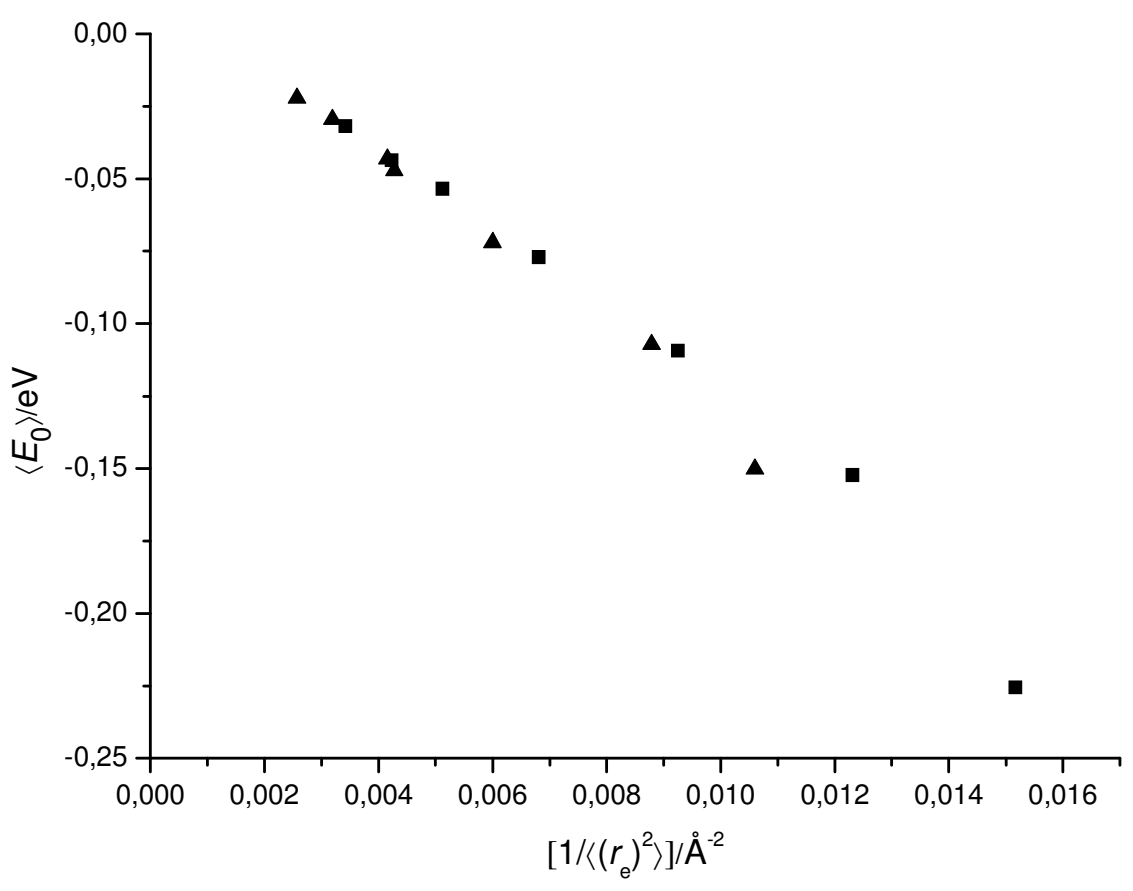


Figure 7. Turi, Madarász, and Rossky

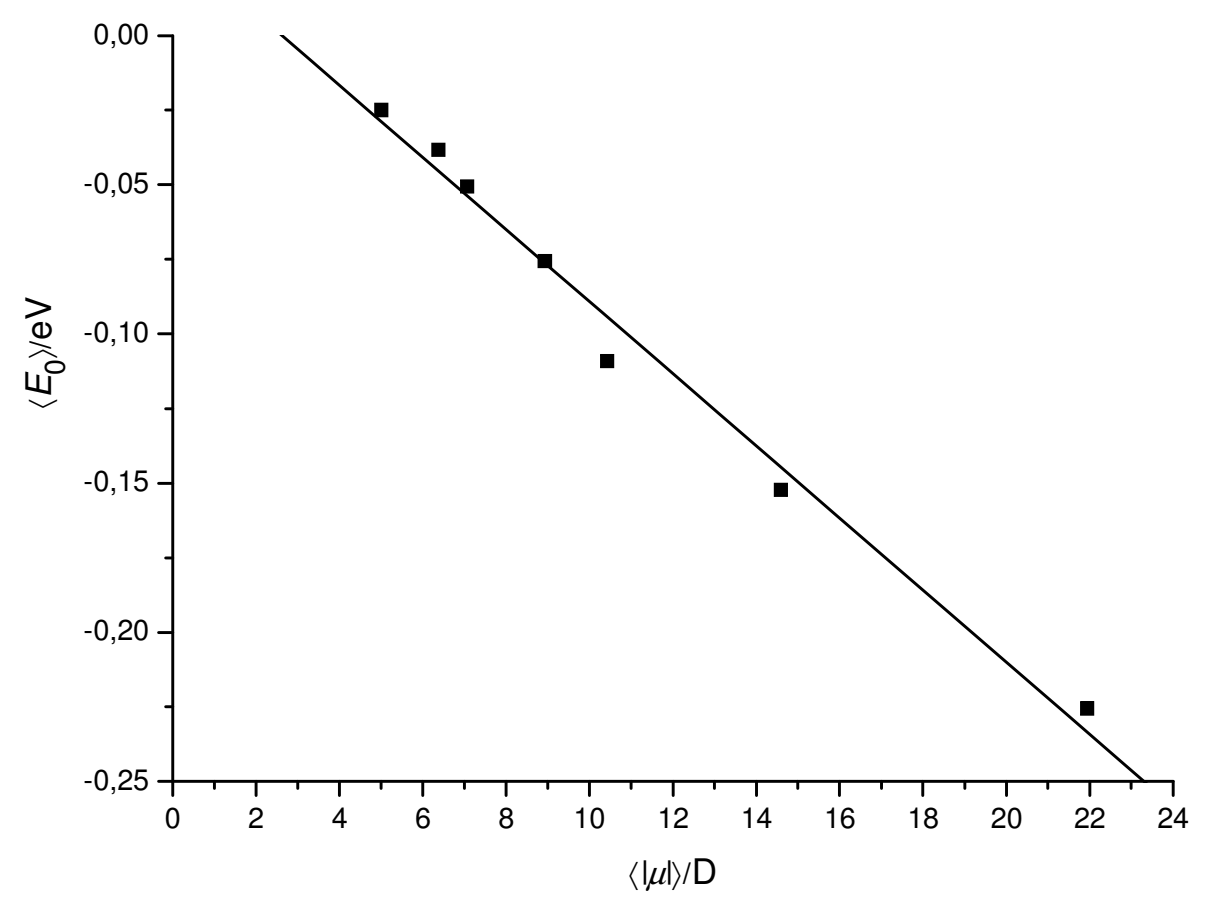


Figure 8. Turi, Madarász, and Rossky

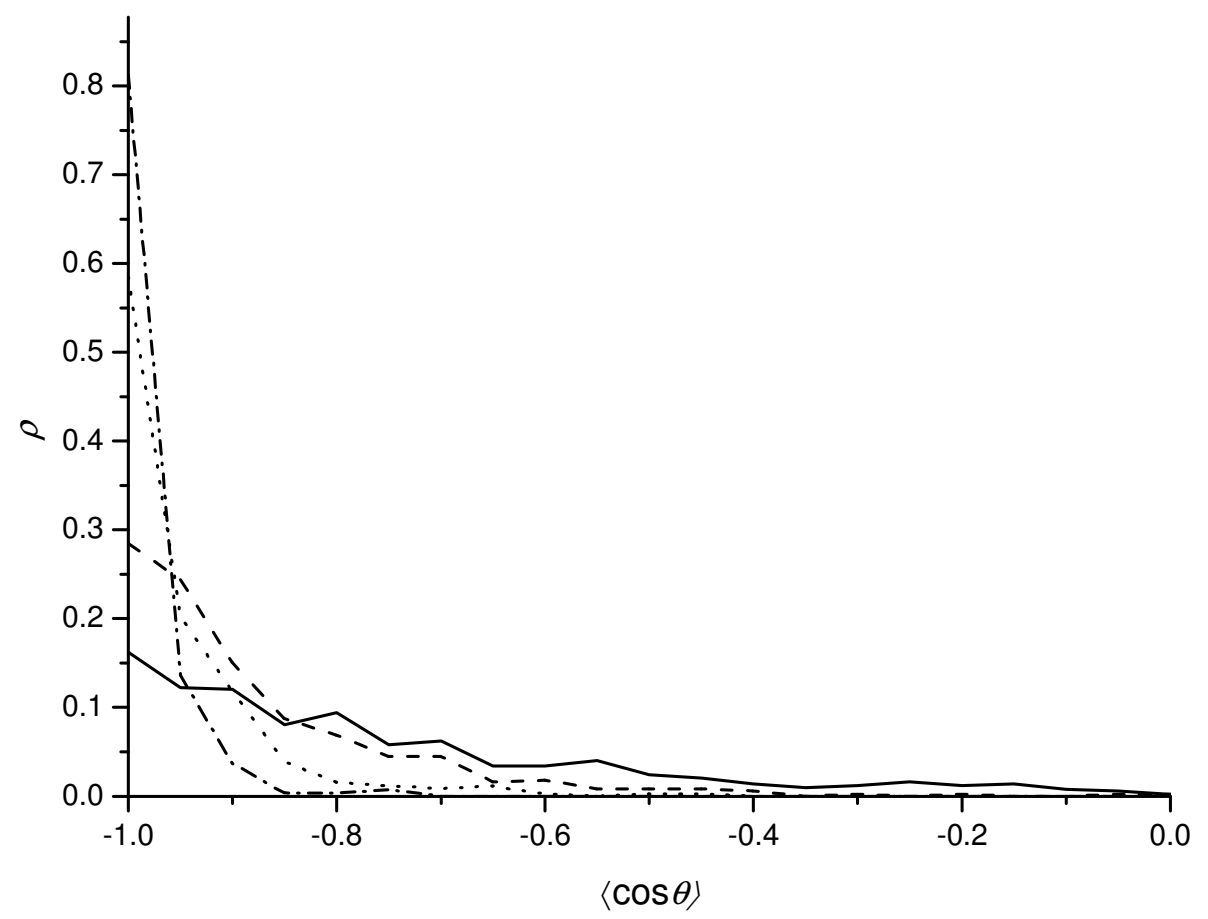


Figure 9. Turi, Madarász, and Rossky

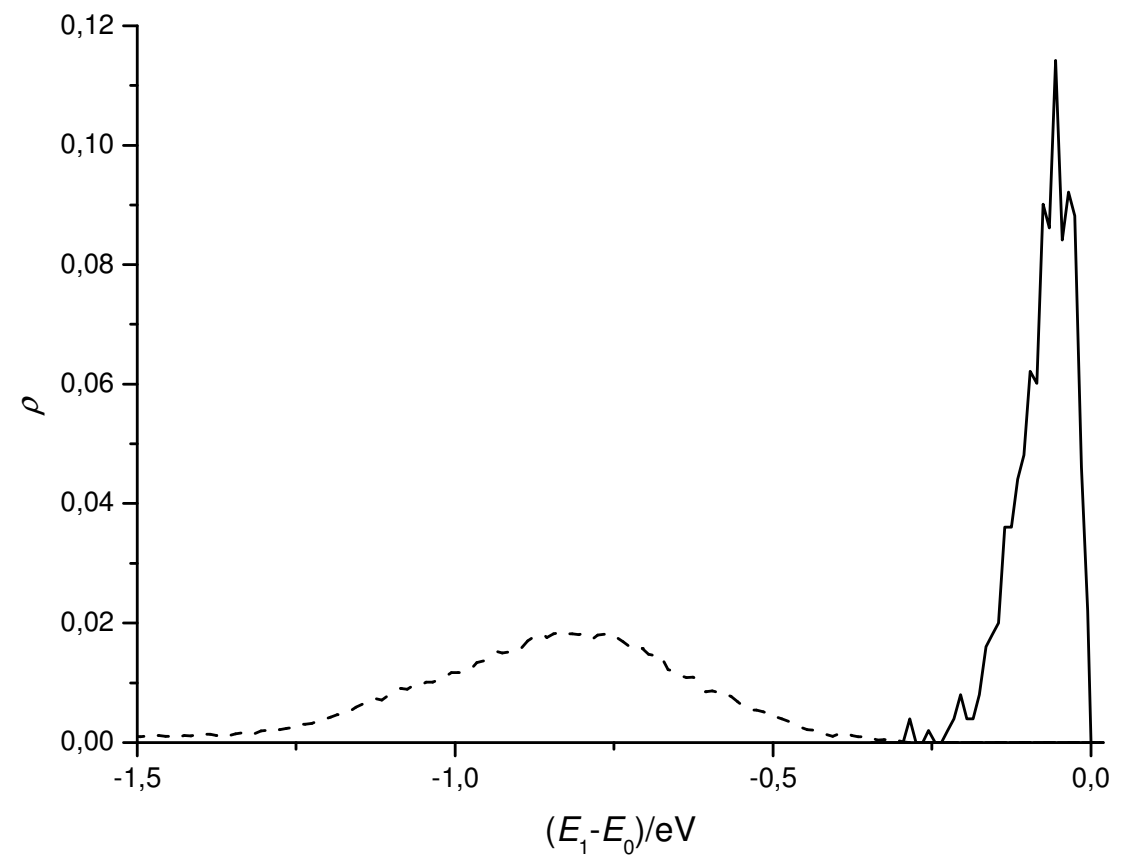




\section{References}

${ }^{1}$ H. Haberland, H. G. Schindler and D. R. Worsnop, Ber. Bunsenges. Phys. Chem. 88, 270 (1984).

${ }^{2}$ H. Haberland, C. Ludewigt, H. G. Schindler and D. R. Worsnop, J. Chem. Phys. 81, 3742 (1984).

${ }^{3}$ E. J. Hart and J. W. Boag, J. Am. Chem. Soc. 84, 4090 (1962).

${ }^{4}$ C. Garrett, D. A. Dixon, D. M. Camaioni, D. M. Chipman, M. A. Johnson, C. D. Jonah, G. A. Kimmel, J. H. Miller, T. N. Rescigno, P. J. Rossky, S. S. Xantheas, S. D. Colson, A. H. Laufer, D. Ray, P. F. Barbara, D. M. Bartels, K. H. Becker, K. H. Bowen, Jr., S. E. Bradforth, I. Carmichael, J. V. Coe, L. R. Corrales, J. P. Cowin, M. Dupuis, K. B. Eisenthal, J. A. Franz, M. S. Gutowski, K. D. Jordan, B. D. Kay, J. A. LaVerne, S. V. Lymar, T. E. Madey, C. W. McCurdy, D. Meisel, S. Mukamel, A. R. Nilsson, T. M. Orlando, N. G. Petrik, S. M. Pimblott, J. R. Rustad, G. K. Schenter, S. J. Singer, A. Tokmakoff, L.-S. Wang, C. Wittig, and T. S. Zwier, Chem. Rev. 105, 355 (2005). ${ }^{5}$ J. V. Coe, G. H. Lee, J. G. Eaton, S. T. Arnold, H. W. Sarkas, and K. H. Bowen, J. Chem. Phys. 92, 3980 (1990).

${ }^{6}$ R. N. Barnett, U. Landman, C. L. Cleveland, and J. Jortner, Phys. Rev. Lett. 59, 811 (1987).

${ }^{7}$ R. N. Barnett, U. Landman, C. L. Cleveland, and J. Jortner, J. Chem. Phys. 88, 4429 (1988).

${ }^{8}$ J. Schnitker, and P. J. Rossky, J. Chem. Phys. 86, 3471 (1987).

${ }^{9}$ P. J. Rossky, and J. Schnitker, J. Phys. Chem. 92, 4277 (1988).

${ }^{10}$ P. Ayotte, and M. A. Johnson, J. Chem. Phys. 106, 811 (1997). 
${ }^{11}$ J. V. Coe, A. D. Earhart, M. H. Cohen, G. J. Hoffman, H. W. Sarkas, and K. H. Bowen, J. Chem. Phys. 107, 6023 (1997).

12 J. V.Coe, Int. Rev. Phys. Chem. 20, 33 (2001).

${ }^{13}$ D. M. Bartels, J. Chem. Phys. 115, 4404 (2001).

${ }^{14}$ G. Makov, and A. Nitzan, J. Phys. Chem. 98, 3459 (1994).

${ }^{15}$ A. E. Bragg, J. R. R. Verlet, A. Kammrath, O. Cheshnovsky, and D. M. Neumark, Science 306, 669 (2004).

${ }^{16}$ D. H. Paik, I-R. Lee, D.-S. Yang, J. S. Baskin, and A. H. Zewail, Science 306, 672 (2004).

${ }^{17}$ N. I. Hammer, J.-W. Shin, J. M. Headrick, E. G. Diken, J. R. Roscioli, G. H. Weddle, and M. A. Johnson, Science 306, 675 (2004).

${ }^{18}$ N. I. Hammer, J. R. Roscioli, and M. A. Johnson, J. Phys. Chem. A 109, 7896 (2005).

${ }^{19}$ J. R. R. Verlet, A. E. Bragg, A. Kammrath, O. Cheshnovsky, and D. M. Neumark, Science 307, 93 (2005).

${ }^{20}$ J. R. R. Verlet, A. E. Bragg, A. Kammrath, O. Cheshnovsky, and D. M. Neumark, Science 310, 1769 (2005).

${ }^{21}$ H. M. Lee, S. Lee, and K. S. Kim, J. Chem. Phys. 119, 187 (2003).

${ }^{22}$ K. Wang, and K. D. Jordan, J. Chem. Phys. 119, 11645 (2003).

${ }^{23}$ A. Khan, J. Chem. Phys. 121, 280 (2004).

${ }^{24}$ J. M. Herbert, and M. Head-Gordon, J. Phys. Chem. A 109, 5217 (2005).

${ }^{25}$ J. M. Herbert, and M. Head-Gordon, Phys. Chem. Chem. Phys. 8, 68 (2006).

${ }^{26}$ L. Turi, W.-S. Sheu, and P. J. Rossky, Science 309, 917 (2005).

${ }^{27}$ L. Turi, W.-S. Sheu, and P. J. Rossky, Science 310, 1769 (2005). 
${ }^{28}$ M. Knapp, O. Echt, D. Kreisle, and E. Recknagel, J. Chem. Phys. 85, 636 (1986).

${ }^{29}$ M. Knapp, O. Echt, D. Kreisle, and E. Recknagel, J. Phys. Chem. 91, 2601 (1987).

${ }^{30}$ K. Toukan, and A. Rahman, Phys. Rev. B 31, 2643 (1985).

${ }^{31}$ M. P. Allen, and D. J. Tildesley, Computer Simulation of Liquids (Clarendon, Oxford, 1987).

${ }^{32}$ L. Turi, M.-P. Gaigeot, N. Levy, and D. Borgis, J. Chem. Phys. 114, 7054 (2001).

${ }^{33}$ L. Turi, and D. Borgis, J. Chem. Phys. 117, 6186 (2002).

${ }^{34}$ R. Spezia, C. Nicolas, A. Boutin and R. Vuillemuier, Phys. Rev. Lett. 91, 208304 (2003).

${ }^{35}$ C. Nicolas, A. Boutin, B. Levy and D. Borgis, J. Chem. Phys. 118, 9689 (2003).

${ }^{36}$ J. Rodriguez and D. Laria, J. Phys. Chem. B 109, 6473 (2005).

${ }^{37}$ F. Wang, and K. D. Jordan, Ann. Rev. Phys. Chem. 54, 367 (2003).

${ }^{38}$ K. D. Jordan, Science 306, 618 (2004).

${ }^{39}$ F. A. Webster, P. J. Rossky and R. A. Friesner, Comput. Phys. Commun. 63, 494 (1991).

${ }^{40}$ K. A. Motakabbir, and P. J. Rossky, Chem. Phys. 129, 253 (1989).

${ }^{41}$ K. A. Motakabbir, J. Schnitker, and P. J. Rossky, J. Chem. Phys. 97, 2055 (1992).

${ }^{42}$ J. Schnitker, and P. J. Rossky, J. Chem. Phys. 86, 3462 (1987).

${ }^{43}$ Gaussian 03, Revision C.02, M. J. Frisch, G. W. Trucks, H. B. Schlegel, G. E. Scuseria, M. A. Robb, J. R. Cheeseman, J. A. Montgomery, Jr., T. Vreven, K. N. Kudin, J. C. Burant, J. M. Millam, S. S. Iyengar, J. Tomasi, V. Barone, B. Mennucci, M. Cossi, G. Scalmani, N. Rega, G. A. Petersson, H. Nakatsuji, M. Hada, M. Ehara, K. Toyota, R. Fukuda, J. Hasegawa, M. Ishida, T. Nakajima, Y. Honda, O. Kitao, H. Nakai, M. Klene, 
X. Li, J. E. Knox, H. P. Hratchian, J. B. Cross, V. Bakken, C. Adamo, J. Jaramillo, R. Gomperts, R. E. Stratmann, O. Yazyev, A. J. Austin, R. Cammi, C. Pomelli, J. W. Ochterski, P. Y. Ayala, K. Morokuma, G. A. Voth, P. Salvador, J. J. Dannenberg, V. G. Zakrzewski, S. Dapprich, A. D. Daniels, M. C. Strain, O. Farkas, D. K. Malick, A. D. Rabuck, K. Raghavachari, J. B. Foresman, J. V. Ortiz, Q. Cui, A. G. Baboul, S. Clifford, J. Cioslowski, B. B. Stefanov, G. Liu, A. Liashenko, P. Piskorz, I. Komaromi, R. L. Martin, D. J. Fox, T. Keith, M. A. Al-Laham, C. Y. Peng, A. Nanayakkara, M. Challacombe, P. M. W. Gill, B. Johnson, W. Chen, M. W. Wong, C. Gonzalez, and J. A. Pople, Gaussian, Inc., Wallingford CT, 2004.

${ }^{45}$ P. R. Antoniewicz, G. T. Bennett, and J. C. Thompson, J. Chem. Phys. 77, 4573 (1982).

${ }^{46} \mathrm{~L}$. Turi manuscript in preparation. 